\title{
Peripheral sea-fan neovascularization as the initial manifestation of systemic lupus erythematosus retinopathy
}

\begin{abstract}
Purpose: To Describe a case of Sea-fan Neovascularization in a SLE patient.

Method: Case report

A 35 year old female who underwent a right hemicolectomy for gangrene gut of undetermined cause was referred to the Ophthalmology department for fundus examination. A Complete ocular examination along with Fluorescein Angiography was done. Fundus examination revealed neovascularization in the temporal quadrant of her right eye. Fluorescein angiography revealed a sea-fan neovascularization corresponding to the neovascular tuft. $\mathrm{Hb}$ electrophoresis revealed no abnormal hemoglobin. ANA and anti-dsDNA tests were positive. She was referred to the immunology department where she was diagnosed as a case of SLE according to the SLE diagnostic criteria of the American Rheumatology Association.

Conclusion: Sea-fan neovascularization can be seen in SLE. To the best of our knowledge this is the first time sea-fan neovascularization is described in a case of SLE. It also demonstrates the diagnostic difficulty of SLE when it presents without the usual manifestations and the importance of a thorough Ophthalmological evaluation in such patients.
\end{abstract}

Keywords: sea-fan retinopathy, SLE
Volume 3 Issue 3 - 2015

Vignesh AP, Renuka Srinivasan
Department of Ophthalmology, JIPMER, India

Correspondence: Vignesh AP, Department of Ophthalmology, JIPMER, No 12 Kamaraj street tagorenagar Pondicherry, India, Tel 919894910033, Email apvicky2010@gmail.com

Received: October 25, 2015 | Published: November 13, 2015

\section{Introduction}

SLE is a chronic autoimmune disease and an important cause of systemic vasculitis. Ophthalmic manifestations range from dry eye to optic neuritis. In the retina it usually causes retinal hemorrhages and vascular occlusions which leads to vision loss. ${ }^{1}$

\section{Case report}

A 35 year old female who underwent a right hemicolectomy for gangrene gut of undetermined cause was referred to the Ophthalmology department for fundus examination. There was no history of floaters or diminution of vision. She had systemic complaints of fatigue, joint pain. On examination, her visual acuity was $6 / 6$ in both the eyes. Anterior segment examination revealed no abnormalities. Dilated fundus examination revealed neovascularization in the temporal quadrant of her right eye without gross evidence of vasculitis (Figure 1). Intraocular pressure in both the eyes by applanation method was 14 and $15 \mathrm{~mm} \mathrm{Hg}$ respectively. Co-morbid conditions like hypertension, diabetes or any cardiac disease were ruled out by doing the necessary investigations. Fluorescein angiography revealed Peripheral sea-fan neovascularization corresponding to the neovascular tuft surrounded by areas of capillary non-perfusion in her right eye (Figure 2). Left eye did not reveal any abnormality in fluorescein angiography. Blood counts were normal. Peripheral smear showed no abnormal cells and ESR was $48 \mathrm{~mm} / \mathrm{h}$. Her Sickling test was negative .Hemoglobin analysis showed a value of $10 \mathrm{~g} / \mathrm{dl}$ and $\mathrm{Hb}$ electrophoresis revealed no abnormal hemoglobin. Blood creatinine and 24 hour urine protein levels were raised. ANA and anti-dsDNA tests were positive. She was referred to the Immunology department where she was diagnosed as a case of SLE according to the SLE diagnostic criteria of the American Rheumatology Association. The patient was treated with laser photocoagulation of the involved segment and the other eye was closely being followed up for neovascular changes. Appropriate systemic treatment was started for SLE to treat the systemic vasculitis.

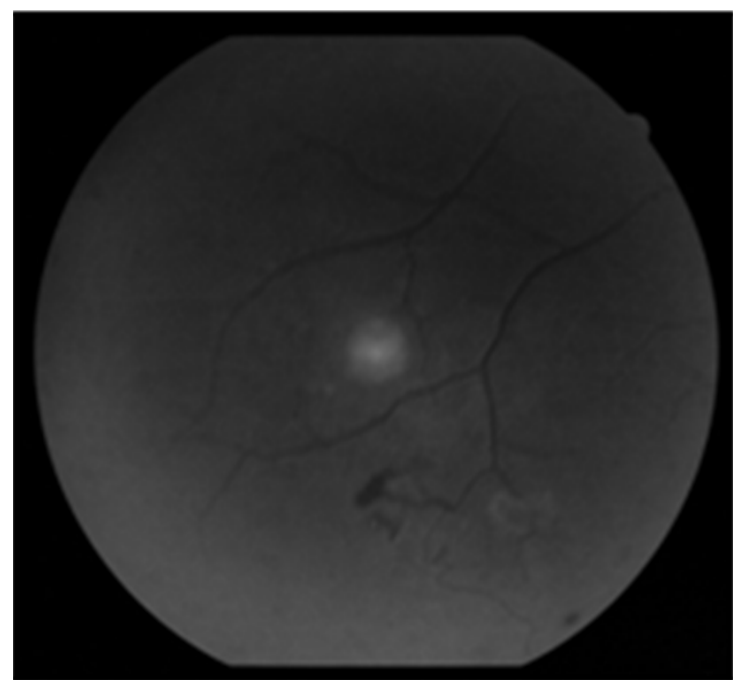

Figure I Red free picture of the neovascular tuft. 


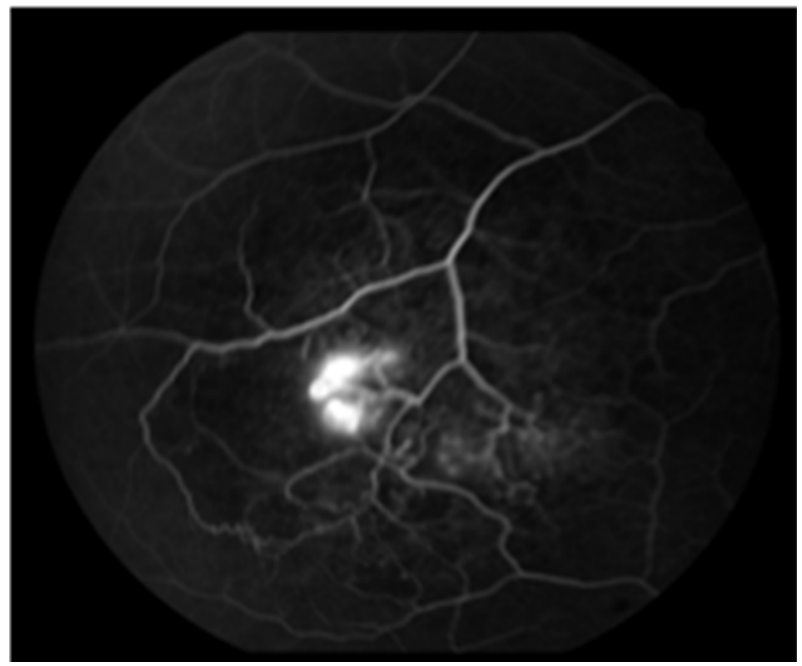

Figure 2 Fluorescein angiography showing sea fan retinopathy.

\section{Discussion}

SLE is a chronic auto-immune disorder involving multiple organ systems. Ocular manifestations are an important component of SLE. The most common ocular manifestation is keratoconjunctivitis sicca and the most common retinal manifestations are cotton wool spots and hemorrhages. ${ }^{1}$ Ocular manifestations are not essential for diagnosis of SLE but offer an important clue to the diagnosis as the retinal vessels can be visualized directly. The onset of SLE retinopathy is usually associated with exacerbation of systemic SLE. In this case the patient did not present with any visual symptoms. The fundus of the patient was quite unremarkable except for the isolated tuft of neovascularization in the right eye. Other causes of sea-fan neovascularization like sickle cell disease, hematological malignancies and vascular disorders were ruled out by normal blood counts, peripheral smear and a normal $\mathrm{Hb}$ electrophoresis. The retinal manifestations of SLE usually consist of cotton wool spots, retinal hemorrhages, vascular occlusions and retinal neovascularization. Sea fan neovascularization has been described with various retinal vasculitis in literature like sickle cell retinopathy, ${ }^{2}$ Eales' disease and hematological malignancies. ${ }^{3,4}$ Ho et al. ${ }^{5}$ reported a rare case of severe vaso-occlusive retinopathy as the primary manifestation in a patient with systemic lupus erythematosus. Zou et $a .^{6}$ reported a case of sequential bilateral central retinal artery occlusion as the primary manifestation of systemic lupus erythematosus. The ocular findings in this case are not characteristic of SLE. This is the first time sea-fan retinopathy is described in SLE. Moreover, it presented as the initial manifestation of SLE retinopathy which further led us to investigate and diagnose the disease. This case also demonstrates the importance of a thorough ocular examination in cases of suspected systemic vascular disorders which can lead a clue to the diagnosis and help in treating the disease at an early stage.

\section{Acknowledgments}

None.

\section{Conflicts of interest}

The author declares there is no conflict of interest.

\section{References}

1. Ostanek L, Modrzejewska M, Bobrowska-Snarska D, et al. Ocular manifestations in patients with systemic lupus erythematosus and antiphospholipid syndrome. Pol Arch Med Wewn. 2014;117(Supple 1):18-23.

2. Freitas LG, Isaac DL, Tannure WT, et al. Retinal manifestations in patients with sickle cell disease referred to a University Eye Hospital. Arq Bras Oftalmol. 2011;74(5):335-337.

3. Macedo MSF, Figueiredo ARM, Ferreira NN, et al. Bilateral Proliferative Retinopathy as the Initial Presentation of Chronic Myeloid Leukemia. Middle East Afr J Ophthalmol. 2013;20(4):353-356.

4. Kim MJ, Yu HG. Case of Bilateral Retinal Neovascularization Associated with Chronic Idiopathic Myelofibrosis. Korean J Ophthalmol. 2010;24(2):131-133.

5. Ho TY, Chung YM, Lee AF, et al. Severe vaso-occlusive retinopathy as the primary manifestation in a patient with systemic lupus erythematosus. J Chin Med Assoc. 2008;71(7):377-380.

6. Zou X, Zhuang Y, Dong FT, et al. Sequential bilateral central retinal artery occlusion as the primary manifestation of systemic lupus erythematosus. Chin Med J (Engl). 2012;125(8):1517-1519. 